\title{
Recomendações de Ações e Tecnologias para a Acessibilidade de Surdos em Curso de Programação a Distância
}

\author{
Márcia Gonçalves de Oliveira ${ }^{1}$, Gabriel Silva Nascimento ${ }^{1}$, \\ Mônica Ferreira Silva Lopes ${ }^{2}$, Anne Carolina Silva ${ }^{2}$, \\ Lucinéia Barbosa da Costa Chagas ${ }^{2}$, Jennifer Gonçalves do Amaral ${ }^{2}$ \\ ${ }^{1}$ Centro de Referência em Formação e Educação a Distância (Cefor) \\ Instituto Federal do Espírito Santo (Ifes) - Vitória - ES \\ ${ }^{2}$ Instituto Federal do Espírito Santo (Ifes) - Campus Serra \\ clickmarcialgmail.com
}

\begin{abstract}
Programming of computers is a complex knowledge because to be learned it demands the operationalization of several skills. In the case of the deaf, programming learning is more difficult in relation to the language domain, whose limitations impact on textual interpretation, problem comprehension, logical sequencing of algorithms, writing and debugging of programs, which are fundamental skills in programming process. Considering the challenges to be overcome to teach programming to the deaf, this work recommends actions and assistive technologies, proposes the extension of a distance programming course from dynamically produced assistive videos in face-to-face teaching and presents reports of experiences of production of assistive videos for a distance programming course.
\end{abstract}

Resumo. A programação de computadores é um conhecimento complexo porque para ser aprendido demanda a operacionalização de várias habilidades. No caso de estudantes surdos, o desafio é ainda maior pois a aprendizagem é dificultada pelas limitações no domínio da segunda língua que impactam diretamente nas habilidades de interpretação textual, na compreensão do problema, na sequenciação lógica de algoritmos, na escrita e na depuração de programas, que são habilidades fundamentais no processo de programação. Considerando os desafios do ensino de programação para surdos, este trabalho recomenda ações e tecnologias assistivas, propõe a extensão de um curso de programação a distância a partir de vídeos assistivos produzidos dinamicamente no ensino presencial e apresenta relatos de experiências de produção de vídeos assistivos para um curso de programação a distância.

\section{Introdução}

Pensar a educação de surdos de um modo geral constitui um dos desafios atuais que vem ganhando cada vez mais espaço nas discussões que permeiam o campo da Educação e da informática nas últimas décadas [Boscarioli et al. 2015, Branco et al. 2017].

Por muito tempo os surdos foram considerados incapazes de aprender, de integrarse à sociedade e de se comunicar. Hoje, porém, a presença de tradutores e intérpretes tornou-se obrigatória em todas as modalidades de ensino, bem como o ensino da Libras 
VII Congresso Brasileiro de Informática na Educação (CBIE 2018)

Anais do XXIV Workshop de Informática na Escola (WIE 2018)

em cursos de Licenciatura e na difusão de informações que possibilitem a entrada de surdos nos espaços públicos e educacionais. É nesse contexto que situamos o ensino de programação para surdos visando discutir os desafios de ensinar por meio da língua de sinais e de uma metodologia mais visual as competências básicas para programar.

Entre as dificuldades enfrentadas por alunos ouvintes na aquisição das competências básicas requeridas na aprendizagem da programação, [Pimentel et al. 2003] delimitam três eixos: (A) Noções de sintaxe e semântica na compreensão de problemas e enunciados; (B) Concepção e formulação de algoritmos; (C) Lógica para a programação.

$\mathrm{O}$ Eixo A diz respeito estritamente às questões da linguagem, bem como o domínio e compreensão da língua em uso nas proposições e capacidade de interpretação. Além disso, influi diretamente nos eixos $B$ e $C$, considerando os algoritmos como instruções sequenciadas que serão aplicadas na execução de comandos e programas que, por sua vez, dependem de relações lógicas preestabelecidas.

Faz-se necessário, portanto, pensar uma metodologia de ensino que perpasse a questão visual dos surdos estimulando o pensamento lógico através da Libras e respeitando suas estruturas de organização semântica e sintática em softwares de programação. A partir daí, apresentam-se novos caminhos para promover o ensino da programação de um modo mais intuitivo que independa do esforço contínuo dos surdos em traduzir primeiro as proposições para então aplicá-las na resolução dos problemas.

Buscando esses novos caminhos, este trabalho apresenta um estudo sobre os desafios dos surdos aprenderem programação, discute ações e tecnologias assistivas para a inclusão de surdos no ensino de programação, propõe a extensão de um curso de programação a distância a partir de vídeos assistivos dinamicamente produzidos em aulas presenciais de programação e apresenta relatos de experiências que resultaram na produção de vídeos assistivos para um curso de programação a distância.

\section{Ações e tecnologias assistivas para inclusão de surdos na programação}

De acordo com [Boscarioli et al. 2015], metodologias adequadas, disponibilização de recursos, assistência pedagógica e psicológica devem ser conhecidas e disponibilizadas para que haja uma efetiva inclusão de surdos na computação. Além disso, as ações voltadas para a inclusão de surdos no domínio da programação devem ter grande envolvimento entre intérpretes, professores, alunos surdos e alunos ouvintes [Boscarioli et al. 2015]. Nesse trabalho colaborativo, deve-se revisar estratégias de ensino, promover a seleção e o uso adequado de tecnologias que facilitem a comunicação e a colaboração.

Na Tabela 1, destacamos as ações de compreender as limitações dos surdos; de vencer os desafios de comunicação impostos pela linguagem falada, escrita e programada; de capacitar professores e intérpretes para o trabalho colaborativo; e de desenvolver tecnologias de apoio à aprendizagem de programação.

\subsection{Tecnologias assistivas para o ensino e aprendizagem de programação}

Embora poucas soluções tecnológicas tenham sido desenvolvidas para a inclusão de surdos no ensino e aprendizagem de programação, algumas tecnologias têm se mostrado promissoras em áreas da computação como, por exemplo: e-learning, realidade virtual e realidade mista [Abuzinadah et al. 2017]. 
VII Congresso Brasileiro de Informática na Educação (CBIE 2018)

Anais do XXIV Workshop de Informática na Escola (WIE 2018)

Tabela 1. Ações para inclusão de surdos na disciplina de programação

\begin{tabular}{|l|l|}
\hline \hline Referência & Ações \\
\hline [Santos et al. 2011] & Desenvolvimento de um Dicionário Bilíngue em português e LIBRAS. \\
\hline [Boscarioli et al. 2015] & $\begin{array}{l}\text { Treinamento de intérpretes para lidar com situações que ultrapassam a interpretação de sinais já } \\
\text { existentes, atuando na criação de sinais específicos que enriquecem o portfólio de sinais }\end{array}$ \\
\hline [Souza Santos et al. 2013a] & $\begin{array}{l}\text { Concepção, construção, avaliação e validação de uma linguagem de programação para o estudo de } \\
\text { lógica de programação por deficientes auditivos, apoiado por uma IDE projetada para dar suporte } \\
\text { às atividades, por intermédio de um intérprete virtual. }\end{array}$ \\
\hline [Santos et al. 2014] & $\begin{array}{l}\text { Desenvolvimento de um ambiente de edição de textos com foco no auxílio a estudantes com de- } \\
\text { ficiência auditiva. Estudam-se também formas de integrar a ferramenta com ambientes de redes } \\
\text { sociais como o Twitter. }\end{array}$ \\
\hline [Gonçalves et al. 2015] & $\begin{array}{l}\text { Produção de Videoaulas de Programação em Java Acessíveis no Contexto de um Projeto de } \\
\text { Capacitação Profissional para Pessoas surdas em três etapas: elaborar conteúdo didático para } \\
\text { gravação, produzir videoaula e gerar versão de produção. }\end{array}$ \\
\hline [Abuzinadah et al. 2017] & $\begin{array}{l}\text { Avaliação da capacidade dos estudantes surdos para estudar e compreender um assunto altamente } \\
\text { técnico como a programação de computadores e a ação de desafiar a percepção generalizada de } \\
\text { que o surdo não pode aprender assuntos complexos. }\end{array}$ \\
\hline \hline
\end{tabular}

Para a aprendizagem de programação, a proposta de [Gallert et al. 2010] é desenvolver um sistema que auxilie os surdos na aprendizagem de algoritmos que permita que desenvolvam programas de computador que utilizem uma linguagem própria com uso da língua de sinais.

Na Tabela 2, apresentamos outras tecnologias que podem ser utilizadas no processo de ensino e de aprendizagem de programação para a inclusão de estudantes surdos.

Além dessas tecnologias, muitos aplicativos estão disponíveis na web e são acessíveis a surdos como, por exemplo, o Whattsapp para surdos, o HandTalk, o ProDeaf e o Youtube. Essas ferramentas podem ser utilizadas no processo de ensino e de aprendizagem de programação para facilitar a comunicação e o trabalho colaborativo entre professores, estudantes surdos e estudantes ouvintes.

\section{Recomendações para o ensino de programação com inclusão de surdos}

De acordo com [Boscarioli et al. 2015], para ter um ensino de fato inclusivo de estudantes surdos, um professor deve conhecer a surdez e como se dá o processo de aquisição de conhecimento dos surdos, utilizar metodologias adequadas com ênfase em aspectos visuais e manter contínua comunicação com o intérprete e com o aluno surdo para avaliar ambiguidades e erros no processo de tradução.

No caso de disciplinas baseadas em tecnologias, como é o caso da programação, os professores, conforme [Krutz et al. 2015], devem trabalhar mais de perto com os intérpretes para apoiar o desenvolvimento de terminologias de domínios que contenham jargões específicos. Outras tecnologias que podem ser utilizadas são o Whattsapp, o Google docs e o Youtube, que são tecnologias que já possuem recursos acessíveis e são utilizados frequentemente pela comunidade surda.

Além dessas tecnologias, os softwares que permitem comunicação em vídeo e videoconferência como Oovoo são os mais comuns e atualmente os que surdos estão usando com mais frequência para comunicação em vídeo são: Facebook, Skype, e Imo. 
VII Congresso Brasileiro de Informática na Educação (CBIE 2018)

Anais do XXIV Workshop de Informática na Escola (WIE 2018)

Tabela 2. Tecnologias Assistivas para o Ensino de Programação

\begin{tabular}{|l|l|l|}
\hline \hline Referência & Tecnologias & Descrição \\
\hline [Oliveira 2012] & Prodeaf & $\begin{array}{l}\text { Plataforma web que disponibiliza um tradutor, um dicionário } \\
\text { Português Libra, além de uma exclusiva ferramenta para criar } \\
\text { sinais em Libras }\end{array}$ \\
\hline [Rocha et al. 2013] & AssistLibras & Assistente gráfico na construção de sinais 3D em libras \\
\hline [Souza Santos et al. 2013b] & Linguagem Proglib e IDE Hands & $\begin{array}{l}\text { Construção de uma linguagem de programação baseada em LI- } \\
\text { BRAS para o ensino de lógica de programação por deficientes } \\
\text { auditivos. }\end{array}$ \\
\hline [Silva Soares et al. 2014] & Visual JO & $\begin{array}{l}\text { Objeto de aprendizagem acessível, que tem por objetivo o en- } \\
\text { sino de linguagem de programação Java a pessoas com de- } \\
\text { ficiência física e auditiva através do estímulo visual }\end{array}$ \\
\hline [Borges et al. 2015] & $\begin{array}{l}\text { Glossário interativo(texto, fotos e } \\
\text { vídeos) }\end{array}$ & $\begin{array}{l}\text { Glossário interativo de sinais e termos técnicos utilizando mo- } \\
\text { delagem 3D (animação) }\end{array}$ \\
\hline [Pereira and da 2016] & Teclibras & $\begin{array}{l}\text { Protótipo web livre e gratuito, com tradução dos termos } \\
\text { técnicos de informática em libras }\end{array}$ \\
\hline [Santos Sobrinha et al. 2016] & $\begin{array}{l}\text { Plataforma para auxílio ao ensino } \\
\text { de programação e robótica pe- } \\
\text { dagógica }\end{array}$ & $\begin{array}{l}\text { Plataforma permite a construção de programas em Python por } \\
\text { meio da composição de elementos visuais. }\end{array}$ \\
\hline JBranco et al. 2017] & $\begin{array}{l}\text { JLAD (Java learning Object to } \\
\text { Assist the Deaf) }\end{array}$ & $\begin{array}{l}\text { Objeto de aprendizado criado com base nas teorias do apren- } \\
\text { dizado ativo e zona de desenvolvimento proximal (ZDP). Ele } \\
\text { possui um IDE simples e ferramentas de colaboração para a } \\
\text { assistência e um acompanhamento das atividades práticas de } \\
\text { forma remota e assíncrona. Essa colaboração envolve alunos, } \\
\text { tutores e intérpretes de Libras }\end{array}$ \\
\hline [Calé et al. 2017] & LABRASTI & $\begin{array}{l}\text { Ferramenta web colaborativa para que alunos possam sugerir } \\
\text { sinais que ainda não tem tradução para LIBRAS e posterior } \\
\text { votação e discussão das mesmas }\end{array}$ \\
\hline [Branco et al. 2017] & $\begin{array}{l}\text { Aplicativo móvel que exibe os termos sobre Tecnologia da } \\
\text { Informação representados em pequenos vídeos em LIBRAS }\end{array}$ \\
\hline \hline
\end{tabular}

\section{Extensão de curso de programação a distância a partir de vídeos assistivos}

Após a realização do estudo sobre as dificuldades de aprendizagem de surdos, sobre ações e tecnologias assistivas que têm sido aplicadas no processo de ensino e de aprendizagem de programação com a inclusão de surdos, propomos a extensão de um curso de programação a distância a partir de vídeos assistivos Youtube produzidos em aulas presenciais de programação.

Para produzir esses vídeos assistivos, formamos uma equipe composta por duas professoras de programação, uma professora de matemática, dois intérpretes de libras e estudantes de iniciação científica atuando como tutores. Essa equipe está desenvolvendo o curso, iniciando com as recomendações de [Gonçalves et al. 2015], definidas na sequência a seguir:

1. Criar um glossário técnico com os termos da linguagem a ser ensinada

2. Realizar aulas presenciais para levantar características específicas e necessidades inerentes ao ensino de surdos e para gerar videos os de programação.

3. Planejar e discutir com o intérprete a linguagem dos conteúdos da aula.

4. Familiarizar-se com a cultura surda através de livros, filmes, oficinas e palestras.

5. Como os surdos olham para o intérprete, recomenda-se, no vídeo, o professor aparecer na abertura e no fechamento, mas que no desenrolar da aula apareçam só os slides e o intérprete. 
VII Congresso Brasileiro de Informática na Educação (CBIE 2018)

Anais do XXIV Workshop de Informática na Escola (WIE 2018)

6. Criar legendas em português para facilitar a compreensão daqueles que ficaram surdos e têm dificuldades com Libras.

7. Criar uma playlist de vídeos com partes da aula presencial para a aula a distância.

8. Registrar as lições aprendidas

Os vídeos assistivos foram planejadas e adaptadas conforme necessidades dos estudantes surdos, poderão ser utilizadas junto com o glossário técnico e as lições aprendidas como materiais de um curso a distância de programação com inclusão de estudantes surdos. Dessa forma, a partir dos videos assistivos gerados no ensino presencial, estendemos dinamicamente um curso de programação a distância acessível para surdos.

A vantagem de produzir vídeos assistivos é que estes poderão ser produzidos uma só vez na modalidade presencial e reproduzido várias vezes na modalidade a distância.

\section{Relatos de Experiências}

Em uma experiência com um aluno de Ciência da Computação surdo, foram observadas as seguintes dificuldades: compreender os enunciados dos problemas e convertê-los para a linguagem algorítmica, mesmo com o auxílio de intérprete [Boscarioli et al. 2015]. Além disso, conforme [Boscarioli et al. 2015], há uma impossibilidade de aferir se um determinado conceito foi corretamente assimilado pelo aluno quando o intérprete não tem formação na área e o professor não conhece Libras.

Considerando essas dificuldades, iniciamos a proposta metodológica deste trabalho de produzir vídeos assistivos para um curso de programação a distância a partir de aulas presenciais de programação para estudantes surdos. Neste trabalho, produzimos vídeos a partir de duas experiências de ensino de programação, dentre os quais destacamos dois: um experimental para avaliação diagnóstica de uma aula de programação para surdos e outro aplicando parte da proposta metodológica de produção de vídeo assistivo.

Na produção do primeiro vídeo na Experiência 1, participaram uma professora de programação que não sabe Libras, um intérprete de Libras com conhecimento básico de programação, duas estudantes surdas e duas estudantes ouvintes de iniciação científica que atuaram como observadoras da aula.

Os conteúdos da aula eram de introdução à programação e de apresentação dos principais conceitos. Nessa experiência, a aula foi planejada pela professora como outras aulas de programação, mas com interpretação de Libras e aplicando uma abordagem mais pragmática considerando as limitações de domínio da linguagem das estudantes surdas.

Nessa primeira experiência, não houve um planejamento conjunto com o intérprete de libras e não foi criado um dicionário com os termos técnicos. E a aula tinha como objetivo apenas identificar necessidades de aprendizagem das alunas surdas e reorientar o ensino conforme essas necessidades.

Já na produção do vídeo da Experiência 2, participaram uma professora de lógica matemática com conhecimentos básicos de Libras, a professora de programação, uma estudante surda e o intérprete da Experiência 1, um intérprete de Libras sem conhecimentos prévios de lógica e de programação e um professor de Libras, que é surdo. 
VII Congresso Brasileiro de Informática na Educação (CBIE 2018)

Anais do XXIV Workshop de Informática na Escola (WIE 2018)

\subsection{Experiência 1}

Iniciada a aula e a sua gravação audiovisual, antes de entrar nos conceitos de programação, a professora desenvolveu interagindo com as alunas surdas um programa em Linguagem Python por meio da ferramenta Python Tutor. A decisão de iniciar a aula com um exemplo prático e não com os conceitos essenciais de programação como em outras aulas foi para que os conceitos fossem aprendidos de forma prática, uma vez que as dificuldades de domínio da língua poderiam interferir na compreensão de conceitos mais abstratos.

O exemplo prático consistiu de um programa para somar dois números e exibir em tela o resultado. Os objetivos eram apresentar uma aplicação útil da programação e reforçar o entendimento da sequência lógica para resolver esse problema.

Ao explicar o programa em Python, foram introduzidos os conceitos de memória (variáveis), atribuição $(=)$, que significa armazenamento de um valor em memória), operação aritmética de soma $(+)$ e comandos de entrada (input(mensagem)), de saída (print) e de conversão de números escritos em texto para inteiros.

Para explicar esses conceitos de forma prática, utilizamos o Python Tutor ${ }^{1}$ por ser um sistema em nuvem mais simples, visual e didático para interpretação e execução de programas escritos em Python. O código e o funcionamento desse programa no Python Tutor são apresentados na Figura 1.

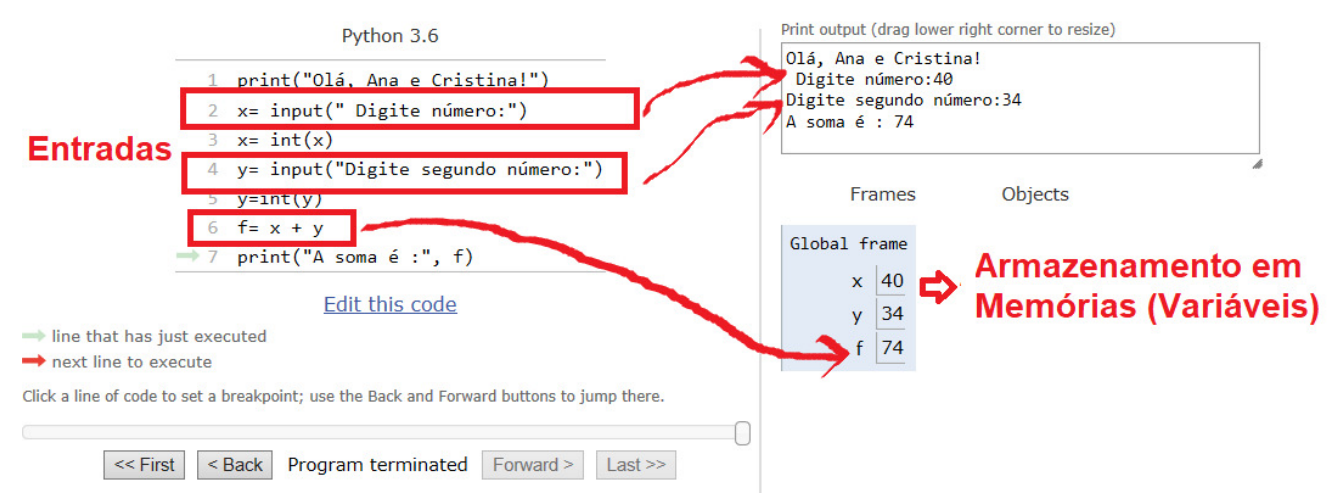

Figura 1. Exemplo de Programa Python explicado no Python Tutor

A principal dificuldade das alunas surdas foi a compreensão da operação de soma com o operador "+". Elas não compreenderam o sinal do intérprete. Em seguida, a professora desenhou no painel da aula o sinal de soma e elas compreenderam a operação após ajudarem-se mutuamente por meio de uma "conversa em Libras". No entanto, o programa só passou a ter significado real para elas quando o problema foi contextualizado à operação de soma de um caixa de supermercados.

Embora o exemplo contemplasse vários conceitos de programação, nessa aula foram trabalhadas principalmentes as habilidades de compreensão e de sequência lógica, reforçando os conceitos de entrada, processamento e saída e a ideia de algoritmo, sempre fazendo alusões ao mundo real. A partir daí, as estudantes foram desafiadas a observar e escrever sequências lógicas de seu cotidiano.

\footnotetext{
${ }^{1}$ Disponível em: pythontutor.com
} 
VII Congresso Brasileiro de Informática na Educação (CBIE 2018)

Anais do XXIV Workshop de Informática na Escola (WIE 2018)

Para a professora de programação, as principais dificuldades dessa primeira experiência foram dar uma aula sem que as alunas olhassem para ela, falar de forma bem pausada, expressar-se de forma mais simples e visual e ter uma sincronia de sua fala com a tradução do intérprete de Libras.

O vídeo assistivo experimental dessa experiência de ensino de programação com interpretação em LIBRAS foi disponibilizado no Youtube ${ }^{2}$.

\subsection{Experiência 2}

Após análise do vídeo resultante da Experiência 1, verificamos quão importante é a interação professor-intérprete no planejamento de aulas de programação. Dessa forma, para produzir os demais vídeos assistivos, identificamos as principais falhas na Experiência 1, realizamos novas oficianas presenciais de programação e produzimos outros vídeos assistivos para futuramente evolui-los para video-aulas de programação, conforme algumas das recomendações de [Gonçalves et al. 2015] na Seção 3.

$\mathrm{Na}$ Experiência 2, destacamos duas oficinas realizadas que contribuíram para uma nova dinâmica da oficina final do curso e do último vídeo assistivo. Essa oficina, que foi a síntese do curso, envolveu as principais habilidades de programação: compreensão do problema, elaboração de sequências lógicas, construção de expressões lógicas e organização de código em estruturas condicionais e de repetição.

A aula de expressões lógicas aconteceu em duas etapas: compreendendo a lógica matemática e construindo sentenças lógicas. A primeira etapa foi planejada junto com um intérprete de Libras que não tinha conhecimentos prévios de lógica e nem de programação e com as professoras de lógica matemática e de programação. A segunda etapa foi realizada da mesma forma que a Experiência 1, mas destacando que o intérprete desenvolveu um conhecimento prévio participando da primeira etapa.

Antes da aula da primeira etapa, a professora de programação destacou as dificuldades que as alunas surdas tiveram na compreensão do sinal de soma e, por isso, poderiam ter dificuldades maiores na compreensão dos operadores lógicos e relacionais. Houve também a orientação de evitar proposições muito abstratas ou com excesso de termos matemáticos. Já o intérprete teve uma atenção maior na tradução/explicação dos sinais lógicos e relacionais em Libras.

Seguindo a Recomendação 1 de [Gonçalves et al. 2015], na primeira etapa, os termos técnicos da aula foram identificados e apresentados ao intérprete de libras para estudo prévio dos sinais de Libras.

A Experiência 1 consolidou a Recomendação 2 por levantar características e necessidades inerentes ao ensino de surdos para a realização das demais oficinas.

Na primeira etapa, o planejamento da aula e as discussões prévias dos conteúdos entre as professoras e o intérprete de Libras contribuíram para a consolidação da Recomendação 3 e a Recomendação 4 foi bem aplicada pelo intérprete, uma vez que este era pesquisador da cultura surda.

O posicionamento da professora de lógica e do intérprete, a forma de olhar e a comunicação de ambos com a estudante surda da experiência contribuíram para uma me-

\footnotetext{
${ }^{2}$ Vídeo da primeira oficina de programação para surdos: https://www.youtube.com/watch?v=QlvxwUWT5MM
} 
VII Congresso Brasileiro de Informática na Educação (CBIE 2018)

Anais do XXIV Workshop de Informática na Escola (WIE 2018)

lhor construção de cenário do vídeo assistivo, conforme a Recomendação 5. Além disso, a preparação de ambos, o conhecimento da cultura surda pelo intérprete e a participação da professora e do intérprete da Experiência 1 como observadores fizeram toda a diferença no desenvolvimento dessa segunda etapa.

Ao final da oficina da primeira etapa, a aluna surda conseguiu completar com rapidez uma tabela-verdade de um exercício de lógica envolvendo os principais operadores lógicos. Para a professora de programação, essa aula prévia de lógica matemática e o vídeo assistivo dela resultante contribuíram muito para a oficina da segunda etapa, que foi a oficina de expressões lógicas e de estruturas de controle condicional if.

$\mathrm{Na}$ segunda etapa, a estudante surda mostrou-se bastante familiarizada com as sentenças lógicas (ou proposições, conforme aprendeu na aula de lógica) e, sozinha, antes que a professora explicasse uma proposição com o operador lógico and, a aluna inferiu a necessidade deste em uma expressão lógica abstrata para avaliar se uma pessoa paga passagem de ônibus, isto é, se ela tem idade entre 50 e 60 anos. Vale destacar que, para construir essa simples expressão, a aluna precisou utilizar operadores lógicos e relacionais, o que já não foi tão difícil compreendê-los quanto foi na compreensão do sinal de soma na Experiência 1.

$\mathrm{Na}$ última oficina do curso, foi realizada, através de um exercício de síntese, uma revisão dos principais conteúdos de programação relacionados às habilidades de compreensão, sequenciação e construção de expressões lógicas. Também foram ensinados os conteúdos de estruturas de controle de repetição. O exercício-síntese foi um programa em Python da urna eleitoral brasileira.

As duas etapas da Experiência 2 contribuíram para um melhoramento na produção dos vídeos assistivos, uma vez que as lições aprendidas e as recomendações seguidas pela professora de programação e pelo intérprete da Experiência 1 resultaram na realização de uma aula final mais interativa e em uma comunicação mais informal e sincronizada entre a professora de programação, o intérprete e a estudante surda.

O vídeo assistivo resultante dessa oficina foi disponibilizado no Youtube ${ }^{3}$.

\subsection{Lições Aprendidas}

Registrando as lições aprendidas que podem melhorar a aprendizagem de programação de surdos, conforme a Recomendação 8, destacamos as seguintes necessidades: planejamento colaborativo da aula com o intérprete de Libras; professores devem compreender o básico de Libras e intérpretes, os termos técnicos da aula que traduzirão para Libras; o desenvolvimento de explicações mais visuais e contextualizadas com o mundo real dos surdos; linguagem simples; sincronia de comunicação entre professor, intérprete e estudantes surdos; conhecimento da cultura surda; mais simplicidade e menos abstrações no desenvolvimento de conteúdos considerando as limitações de domínio da linguagem pelos surdos; e reorientação das práticas pedagógicas para atender necessidades de aprendizagem de estudantes surdos e ouvintes. Considerando essas necessidades, professores e gestores devem ser chamados à reflexão sobre o que de fato estão fazendo em suas práticas educativas para promover a inclusão.

\footnotetext{
${ }^{3}$ Vídeo da última oficina de programação para surdos: https://www.youtube.com/watch?v=CRPIEeC8Jio
} 
VII Congresso Brasileiro de Informática na Educação (CBIE 2018)

Anais do XXIV Workshop de Informática na Escola (WIE 2018)

A criação de legendas em português e da playlist com os vídeos assistivos produzidos em cada aula para inserção no curso de programação a distância, conforme as recomendações 6 e 7, serão os trabalhos futuros.

\section{Considerações finais}

Este trabalho apresentou ações e tecnologias assistivas para o ensino de programação com a inclusão de estudantes surdos. A partir desse estudo, conhecemos os principais desafios e apresentamos recomendações de ações e tecnologias para promover um ensino inclusivo e favorecer a aprendizagem de programação de estudantes surdos. Além disso, propomos um curso de programação estendido da modalidade presencial para a modalidade a distância através da geração de vídeos assistivos.

A produção de cada vídeo assistivo resultou de uma aula de oficina do ensino presencial através de um trabalho colaborativo entre professores e programação, intérpretes de Libras e estudantes surdos.

Após muitos estudos, atualmente, essa metodologia está sendo aplicada no Cefor e apresentamos neste trabalho os primeiros relatos de experiências. Como trabalhos futuros, propomos o relato de todo o processo e apresentação de resultados dessas experiências, que ainda são pioneiras no domínio da aprendizagem de programação.

As principais contribuições deste trabalho para a Informática na Educação são recomendar ações e tecnologias que favoreçam a acessibilidade de surdos no processo de ensino e de aprendizagem de programação e ampliar as possibilidades de formação em programação para pessoas com deficiência auditiva através de vídeos assistivos produzidos dinamicamente a partir de aulas de programação presenciais.

\section{Referências}

Abuzinadah, N. E., Malibari, A. A., and Krause, P. (2017). Towards empowering hearing impaired students' skills in computing and technology. computer, 8(1).

Borges, L. C., Obara, E. N., Leite, C. Z., and Rocha, F. G. (2015). Glossário interativo de libras para a área de computação. Anais do Computer on the Beach, pages 550-551.

Boscarioli, C., Galante, G., Oyamada, M. S., Zara, R. A., and Villwock, R. (2015). Aluno surdo na ciência da computação: Discutindo os desafios da inclusão. In XXIII Anais do Workshop de Informática na Escola (WEI) - CSBC 2015.

Branco, A. C., Maia, P., dos Santos, F., Barros, E., Pinheiro, F. T., Silva, L. C., and do Nascimento, M. (2017). Desafios e experiências no ensino de programação java através de educação a distância para pessoas com deficiência. In Anais do Workshop de Informática na Escola, volume 23, page 1109.

Calé, F. R., Paim, L. N., Volpasso, L. H. S., Benassi, P., Miranda, R., Meneghini, T. L., and Cruz, S. (2017). Librasti: Uma aplicação móvel para levar o vocabulário da tecnologia da informação ao público surdo. XXII ENAPET-Brasília, 6pp.

Gallert, C. S., Guerra, E., and Povala, G. (2010). Sistema de ensino de algoritmos para surdos. Anais do Computer on the Beach, pages 9-10.

Gonçalves, E., Vilela, J., Peixoto, M., Oliveira, F., and Castro, J. (2015). Produção de videoaulas de programação em java acessíveis no contexto de um projeto de capacitação 
VII Congresso Brasileiro de Informática na Educação (CBIE 2018)

Anais do XXIV Workshop de Informática na Escola (WIE 2018)

profissional para pessoas surdas. In Brazilian Symposium on Computers in Education (Simpósio Brasileiro de Informática na Educação-SBIE), volume 26, page 877.

Krutz, D. E., Malachowsky, S. A., Jones, S. D., and Kaplan, J. A. (2015). Enhancing the educational experience for deaf and hard of hearing students in software engineering. In Frontiers in Education Conference (FIE), 2015 IEEE, pages 1-9. IEEE.

Oliveira, J. (2012). Prodeaf: Uma ferramenta colaborativa para a produção de conhecimento em libras. INES.

Pereira, D. F. and da, E. N. (2016). Teclibras: Um protótipo web de apresentação de termos de informática em libras. In Revista Fórum, number 33.

Pimentel, E. P., de França, V. F., Noronha, R. V., and Omar, N. (2003). Avaliação contínua da aprendizagem, das competências e habilidades em programação de computadores. In Anais do Workshop de Informática na Escola, volume 1, pages 533-544.

Rocha, D. F. S., Bittencourt, I. I., and Brito, P. (2013). Assistlibras: Um assistente gráfico para construção de sinais $3 \mathrm{~d}$ da libras. In Anais dos Workshops do Congresso Brasileiro de Informática na Educação, volume 2.

Santos, R. E., Magalhães, C. V., Maciel, J., Correia-Neto, J. S., and Vilar, G. (2014). Informática na educação especial: uma discussão no contexto da educação de surdos. In Brazilian Symposium on Computers in Education (Simpósio Brasileiro de Informática na Educação-SBIE), volume 25, page 622.

Santos, R. E., Magalhães, C. V., Neto, J. S. C., and Júnior, S. S. P. (2011). Proglib: Uma linguagem de programaçao baseada na escrita de libras. In Anais do Workshop de Informática na Escola, volume 1, pages 1533-1542.

Santos Sobrinha, V. H. P., do Nascimento, G. R. A., Gomes, R. D., and Neto, O. d. A. R. (2016). Plataforma para auxílio ao ensino de programação e robótica pedagógica. Revista Principia, 1(31):104-112.

Silva Soares, M. I., Júnior, C. G. F., Silva, L. C., de Oliveira, R. B., Lima, N. A. S., Soares, É. F., et al. (2014). Visual jo2: Um objeto de aprendizagem para o ensino de programação java a deficientes físicos e auditivos através do estímulo visual-um estudo de caso. RENOTE, 12(2).

Souza Santos, R. E., Magalhães, C. V., Correia-Neto, J. S., Queiros, L. M., and Vilar, G. (2013a). Trabalhando lógica de programação com portadores de deficiência auditiva: a experiência com a linguagem proglib e a ide hands. Revista Brasileira de Computação Aplicada, 6(1):32-44.

Souza Santos, R. E., Magalhães, C. V., Correia-Neto, J. S., Queiros, L. M., and Vilar, G. (2013b). Trabalhando lógica de programação com portadores de deficiência auditiva: a experiência com a linguagem proglib e a ide hands. Revista Brasileira de Computação Aplicada, 6(1):32-44. 